\title{
The Formation and Structure of Circumstellar Disks
}

\author{
J.E. Bjorkman \\ Ritter Observatory, Dept. of Physics \& Astronomy, University of \\ Toledo, Toledo, OH 43606-3390, USA
}

\begin{abstract}
Several theories have been proposed to explain the origin of Be star disks. Among them are Wind-Compressed Disks, accretion disks, decretion disks, and "explosive" ejections. In reviewing these mechanisms, I first concentrate on the current status of the Wind-Compressed Disk model. In particular, I discuss how non-radial forces may prevent disk formation and then discuss various physical effects that may restore the disk. Second, I examine the observational evidence and what it tells us about the structure of the disk. Of particular interest is evidence in favor of Keplerian disks. Finally, I discuss theories for Keplerian disk formation and some of the constraints such theories must satisfy.
\end{abstract}

\section{Introduction}

Since the last meeting on Be stars at Boulder in 1986, there has been considerable progress in our understanding of the velocity and density structure of $\mathrm{Be}$ star circumstellar envelopes. For example, we now know that Be stars are surrounded by thin circumstellar disks (K. Bjorkman, these proc.), and these disks appear to have a Keplerian velocity structure (Hummel; Hanuschik, these proc.). Consequently, we must investigate the implications of these observations for the many disk formation theories that have been proposed for Be stars. There are two primary situations where disks occur: infall (accretion), and rapidly rotating outflow. First, we briefly discuss accretion models.

\section{Accretion Disks}

Accretion disks have been studied in the context of active galactic nuclei, cataclysmic variables, and star formation (for a review, see Frank, King, \& Raine 1985). However, the main-sequence lifetime of a $\mathrm{Be}$ star is about $10^{7}-10^{8} \mathrm{yr}$ (Maeder \& Meynet, 1989), so it is unlikely that a remnant protostellar disk could survive for any appreciable time. Thus we expect that only the youngest Be stars could have a remnant protostellar accretion disk. The only other possibility for having an accretion disk is an active mass-transfer binary system.

\subsection{Mass-Transfer Binaries}

In a mass-transfer binary, one star has evolved off the main-sequence to the point at which it fills its Roche lobe. During the subsequent evolution, this 
star (the mass loser) overflows the Roche lobe and the resulting outflow falls down the gravitational potential well toward the other star (the mass gainer). However, owing to the orbital angular momentum of the mass loser, this material (usually) does not fall directly onto the mass gainer but instead goes into orbit, forming a Keplerian accretion disk. Such a scenario has been suggested for many years (e.g., Harmanec \& Kř́iž 1976). In this scenario, the mass gainer will spin-up to critical rotation, naturally explaining the rapid rotation of $\mathrm{Be}$ stars. Although this scenario is certainly happening in some instances, it now appears unlikely that it can explain all Be stars. After examining all the relevant timescales involved, Pols et al. (1991) find that at most $40 \%$ of all Be stars could be produced in mass-transfer systems, while Van Bever \& Vanbeveren (1997) conclude that the upper limit is only $20 \%$ (for an alternative viewpoint, see Gies, these proc.). We conclude that accretion disks must be the minority case, which indicates we need a model of disk formation for isolated (i.e., single) Be stars. In such a situation, the disk must be formed from the stellar outflow.

\section{1-D Equatorial Outflow Models}

Much effort has been expended studying the stellar winds from rapidly rotating hot stars. Although a few 2-D models have been devised (e.g., Poe 1987; Bjorkman \& Cassinelli 1993), most models are really 1-D models, usually of the equatorial outflow (but sometimes they assume the flow occurs at constant latitude; i.e., on the surface of a cone). Classifying these 1-D models according to the main physical driving mechanism, we have:

1. Optically thick line-driven winds. Friend \& Abbott (1986) added rotation to the Castor, Abbott, \& Klein (1975; CAK) model; Lamers and Pauldrach (1991) investigated how von Zeipel (1924) gravity darkening would influence the latitudinal dependence of the outflow, using the bistability mechanism discovered by Pauldrach and Puls (1990).

2. Magnetic fields. Poe \& Friend (1986) added magnetic fields to the CAK model; Chen \& Marlborough (1992) explored weak magnetic fields in slowly accelerating winds; and Koninx (1992) employed Alfvén waves.

3. Acoustic waves. Koninx \& Hearn (1992) also examined whether ordinary pressure (sound) waves could produce the equatorial flow, but discovered the required wave amplitude exceeded the sound speed.

4. Viscous forces. De Araújo \& de Freitas Pacheco (1989) employed viscosity along with an empirical temperature distribution.

5. Empirical forces. Chen, Marlborough, \& Waters (1992) and Marlborough, Chen, \& Waters (1993) empirically determined the force required to produce the observed disk density distribution.

6. Optically thin lines. Chen \& Marlborough (1994); de Araújo, de Freitas Pacheco, \& Petrini (1994); and de Araújo (1995) used an empirical ensemble of optically thin lines to drive a slow equatorial outflow. 
The general problem with most of these models is that there is no physical basis to support the proposed empirical force, or they fail to produce one or more of the following observational constraints: $V_{\text {rot }} \lesssim 0.8 V_{\text {crit }}, \dot{M}_{\text {disk }} \sim 10^{-7} M_{\odot} \mathrm{yr}^{-1}$, and $v_{\infty} \lesssim 100 \mathrm{~km} \mathrm{~s}^{-1}$. More recently, it has become apparent that at least the inner part of the disk is Keplerian with a very small $r$-component of the velocity. Such Keplerian disks cannot be produced with simple radial forces driving the flow, because a Keplerian velocity law requires transport of angular momentum (and radial forces exert no torque). For this reason we must now begin to consider viscous decretion disks.

\subsection{Decretion Disks}

A viscous decretion disk is a variant of the Shakura \& Sunyaev (1973) theory of accretion disks (so-called $\alpha$-disks). Lee, Saio, \& Osaki (1991) used the Shakura $\&$ Sunyaev eddy viscosity (parameterized by $\alpha$ ) to drive a viscous decretion disk, starting from the equator of the star. While such a theory automatically produces a Keplerian disk, the mass-loss rate is determined by an unknown parameter $\alpha$. Furthermore, for such a theory to successfully remove material from the star's equator, the star must be rotating at the critical (break-up) rotation speed, $V_{\text {crit }}$. This would require that all Be stars rotate at the breakup speed, in contradiction to observations. We conclude that some additional mechanism is required to inject material into the disk with enough angular momentum to orbit the star. If such an injection mechanism exists, then the decretion disk theory predicts that the material will spread outward (and inward) forming a viscous decretion disk.

\section{Wind-Compressed Disks}

One method for injecting material into a disk is the Wind-Compressed Disk (WCD) model of Bjorkman \& Cassinelli (1993). Note however, that the model in its basic form does not transport angular momentum, so the WCD will not be Keplerian (again an additional mechanism will be needed). Since many other reviews of the WCD model exist elsewhere (see Bjorkman 1994, 1997), I will simply summarize the basic features.

The fundamental reason a rotating wind produces a disk is that the material tends to orbit the star during the time it is accelerated outward. If the outward acceleration is small compared to the rotation, then the material will orbit down to the equator before it is accelerated outward (recall that the orbital plane is inclined with respect to the equator). After $1 / 4$ of an orbit, it crosses the equator where it meets the corresponding orbit from the opposite (southern) hemisphere. The resulting collision produces a pair of shocks above and below the equator (see Fig. 1), and the subsequent radiative cooling produces a dense disk $\left(\rho_{\text {eq }} / \rho_{\text {pole }} \sim\right.$ $100)$. Note that the dense disk is not a result of enhanced equatorial massloss. Although the equatorial mass-loss is increased by rotation, this is not the primary mechanism. The primary effect of rotation is to redistribute the outflow in latitude (see Fig. 1). Thus the polar circumstellar regions are evacuated while the equatorial regions are enhanced.

The requirement that the orbital motion exceed $1 / 4$ of an orbit implies there is a minimum rotation rate for producing the disk. However, when the rotation 


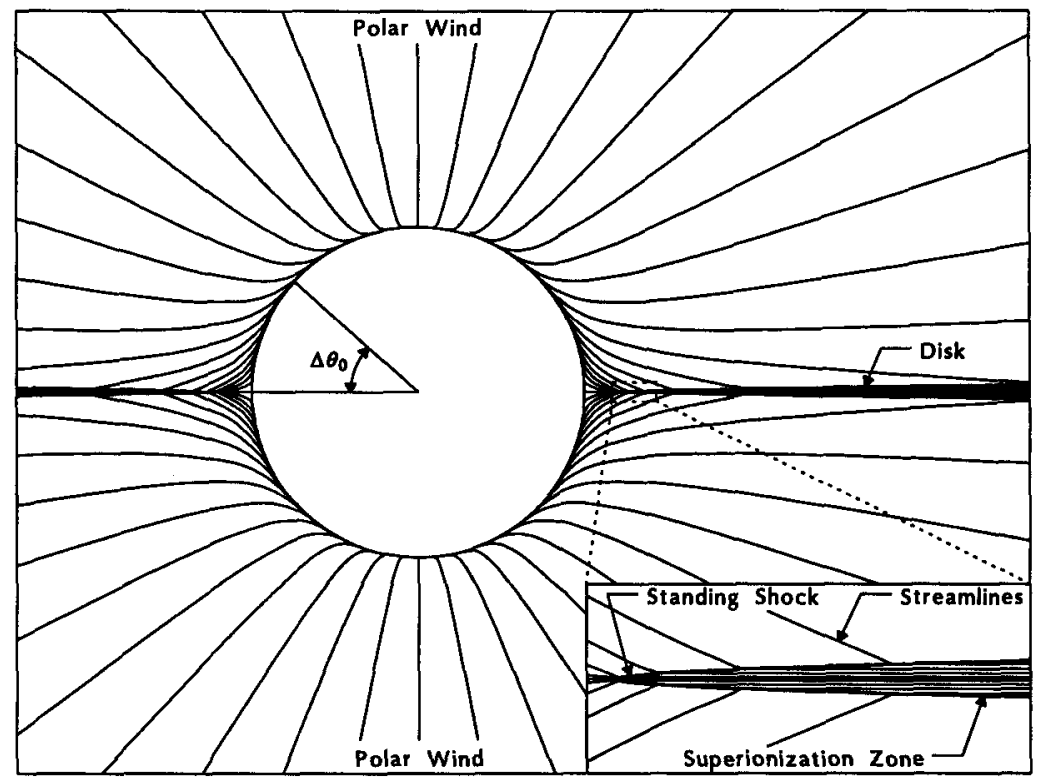

Figure 1. Wind-Compressed Disk. Shown are the streamlines from a rapidly rotating star. Where the streamlines cross the disk, a pair of shocks compresses the flow, and the post-shock cooling produces a dense disk. (Figure from Bjorkman \& Cassinelli 1993.)

rate is less than the disk formation threshold, there can still be equatorial compression without a shock-compressed disk. We call these cases Wind-Compressed Zone (WCZ) models (Ignace, Cassinelli, \& Bjorkman 1996).

\subsection{Ionization Structure}

In the WCD and WCZ models, there is a latitudinal density gradient that produces a corresponding ionization gradient in the wind. Using a NLTE photoionization code (MacFarlane et al. 1993), Bjorkman, Abbott, \& MacFarlane (2000) calculate the ionization structure for a B2.5IV star. Figure 2 shows the ionization distribution of carbon in the stellar wind. Note that C III is the dominant state close to the star, and as the density drops, the X-rays quickly ionize the wind up to $\mathrm{CV}$. However, in the disk $\mathrm{CIII}$ is the dominant state owing to the high disk density. The effects of these ionization fractions on the line profiles may be found in the poster paper by Bjorkman \& Abbott (these proc.). They also find that previous mass-loss rates may have been significantly underestimated as a result of the rapid ionization of the wind.

\subsection{Non-Radial Forces}

The disk formation scenario predicted by the WCD model was verified by Owocki, Cranmer, \& Blondin (1994; OCB) who performed hydrodynamics simulations of a rotating wind. In the original WCD model the radiation force was radial. However, there are small non-radial components in the force vector. 

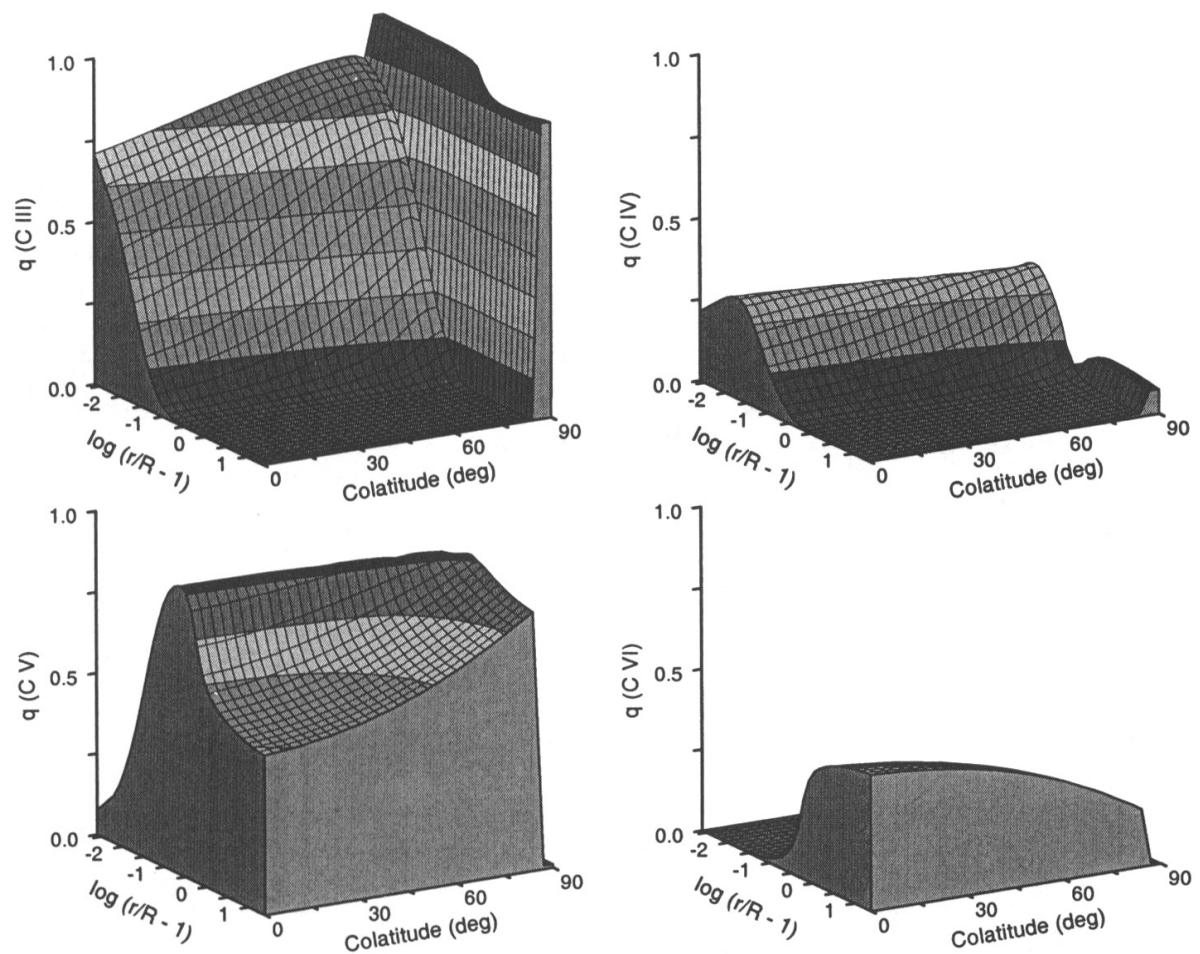

Figure 2. Ionization distribution of carbon. Shown are the ionization fractions of CIII-VI as a function of position.

Owocki, Cranmer, and Gayley (1996; OCG) found that these non-radial components act against disk formation and are large enough to stop the disk formation, producing enhanced polar outflow instead of enhanced equatorial outflow.

The non-radial forces arise from three effects. First, a rapidly rotating star is oblate. The increased equatorial solid angle directs the radiative flux upward away from the equator. Second, the CAK line force depends on the velocity gradient, which is direction-dependent. Since the wind terminal speed increases with latitude, the velocity gradient is larger looking toward the equator than looking toward the pole. The increased velocity gradient produces a larger radiation force, so the equatorial photons produce a larger force than the polar photons, lifting the flow away from the equator. The third effect results from angular momentum conservation, which implies $v_{\phi}$ decreases with radius. Again the CAK force depends on the velocity gradient, producing a retarding torque that rapidly spins-down the flow, decreasing the wind-compression.

The non-radial force calculation performed by OCG employs the Sobolev approximation, using a smoothly accelerating wind. To restore the disk, we must turn off (or greatly reduce) the non-radial forces. Some possibilities include:

1. Optically thin lines. When the lines are optically thin, the Sobolev escape probability $\beta=[1-\exp (-\tau)] / \tau \approx 1$, where $\tau$ is the Sobolev optical depth. 
For a thin line with opacity $k_{L}$, the Sobolev line acceleration,

$$
g_{L}=\frac{2 \pi k_{L}}{c} \int \frac{1-e^{-\tau}}{\tau} I_{\nu}^{*} \mu d \mu \approx \frac{4 \pi k_{L} H_{\nu}}{c}
$$

is proportional to the flux vector, which is radial as long as the star is not rotating very rapidly. Thus we see that the non-radial force components are produced by the optically thick lines. If the wind is driven primarily by thin lines, the non-radial forces will be small. The problem is that to start the wind, there must be at least one optically thick line. Perhaps as a result of the decreasing density, the wind starts with optically thick line-driving and shifts to optically thin lines at larger radii.

2. Clumpy Winds. It is likely the wind instability produces dense clumps in the wind (see Owocki \& Cohen, these proc.). If the velocity gradient across the clump is small, the Sobolev approximation is no longer valid and the Sobolev length is replaced by the physical size of the clump, $l$. In the limit that the clump is optically thick, the radiative acceleration is

$$
g_{\text {clump }}=\frac{4 \pi H}{c} \frac{A_{\mathrm{c}}}{m_{\mathrm{c}}}=\frac{4 \pi H}{c \rho_{\mathrm{c}} l},
$$

where $A_{\mathrm{c}}, m_{\mathrm{c}}$, and $\rho_{\mathrm{c}}$ are the area, mass, and density, respectively, of the clump. Although the lines are optically thick, the force on the clump is radial, unless the star is oblate. Even if the star is oblate, the radiative acceleration will vanish when the mass of the clump is large, leaving gravity as the only force. In this case, the clump will orbit the star, cross the equator, and be added to the disk.

3. Ionization gradients. The disk inhibition calculation assumed constant CAK force multipliers $(k, \alpha, \delta)$ throughout the wind. However there can be rapid increases in the ionization close to the star (see Fig. 2). The rapid increase in ionization will tend to reduce the number of UV lines available to drive the wind, so the non-radial forces may disappear.

Perhaps the most currently promising mechanism for eliminating the non-radial forces is ion decoupling.

\subsection{Ion Decoupling}

The stellar flux drives the wind via line absorption, which is dominated by metal lines, especially in B stars (Abbott 1982). If the density of the wind is large, Coulomb interactions between the metals and the rest of the gas rapidly transfer the momentum gain to the rest of the wind. However when the density is low, the metals decouple from the wind, and the line-driving shuts off outside the decoupling radius (Springmann \& Pauldrach 1992). Porter and Drew (1995) examined ion decoupling in the context of the WCD model and found that it generally enhances wind-compression. If the ion decoupling radius is as small as $1.1 R_{*}$, it effectively shuts off the line-driving (see Fig. 5 of Porter \& Drew). Subsequently Porter \& Skouza (1999) found that a spherically symmetric wind will stagnate and fall back onto the star as a result of ion decoupling. They 
calculate the ion decoupling radius as a function of mass-loss rate and find that the decoupling radius will be less than about $1.1 R_{*}$ when the mass-loss rate is less than $10^{-10} M_{\odot} \mathrm{yr}^{-1}$ (see Fig. 1 of Porter \& Skouza 1999), which is a typical Be star mass-loss rate (Snow 1981; Prinja 1989). Thus we see that ion decoupling will turn off the radiation force at radii greater than $1.1 R_{*}$, so the material will again orbit down to the equator forming a WCD. We conclude that ion decoupling will restore disk formation for stars with weak winds.

\subsection{Observational Constraints}

Unfortunately stars with weak winds have insufficient mass-loss to create a disk dense enough to explain Be stars (Bjorkman 1994). Porter (1997) calculated the IR excess for the WCD model using a typical Be star mass-loss rate and found that the IR excess was two orders of magnitude too small. There are however some stars thought to have disks with much lower densities. Zaal et al. (1997) found weak double-peaked $\mathrm{Br} \alpha$ emission in $2 \mathrm{Vul}$ and HD 203938. The required emission measure corresponds to a disk density two orders of magnitude lower than a typical Be star disk, but consistent with a WCD. Thus, they suggest there may be a class of stars with weak disks.

Although the WCD model provides a natural mechanism for producing a disk, the basic model conserves angular momentum. This is the primary reason the disk density of such models is too small. OCB found that the inner part of the disk falls back onto the star because there is neither sufficient radiation force nor centrifugal force to support the disk. If the basic WCD model could be modified by finding a mechanism to transport angular momentum (such as magnetic fields), then the disk could be rotationally supported. Material would no longer fall back onto the star and a dense disk would result. In such a scenario the WCD mechanism would provide a natural way to feed a Keplerian disk.

\section{Keplerian Disks}

There is now growing evidence, both theoretical and observational, that $\mathrm{Be}$ star disks are Keplerian. The geometry of the disk has been deduced from polarimetry combined with optical interferometry. From interferometry of the $\mathrm{H} \alpha$ emission, Quirrenbach et al. (1997) find that the circumstellar envelopes of Be stars cannot be slightly flattened ellipsoids; they must be thin disks. This enabled Wood, Bjorkman \& Bjorkman (1997) to show that the polarimetry requires that the disk be very thin with an opening angle of about $3^{\circ}$, which is consistent with the scale height of a Keplerian disk (it is also consistent with the thickness of a WCD). Now that we know there is a thin disk, what kind is it: Keplerian, or angular momentum-conserving?

One effective method for studying the disk velocity structure of Be stars is to model the optically thin Fe line emission (Hanuschik 1988, 1995, 1996). He finds that the $r$-component of the velocity must be small (less than the thermal speed) in the region where the Fe lines are formed. Although it is quite difficult to determine the rotational component of the velocity, $v_{\phi}$, Hanuschik, Kozok, \& Kaiser (1988) found $v_{\phi} \sim 1 / r$ (angular momentum conserving), but Mennickent et al. (1994) found $v_{\phi} \sim 1 / r^{1.4}$. Using optical interferometry to constrain the $\mathrm{H} \alpha$ emission radii, Hummel \& Vranken (these proc.) show that 


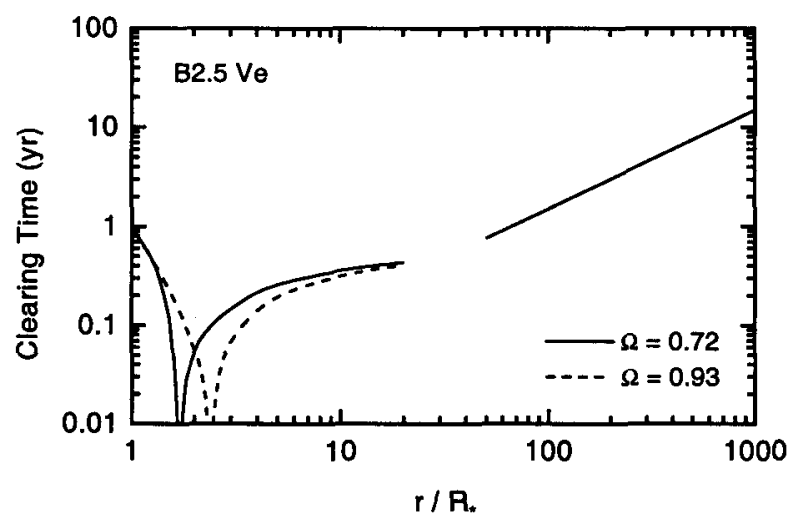

Figure 3. Disk clearing time. The time required for the wind to completely entrain the disk as a function of position is shown for two different rotation rates $\Omega=V_{\text {rot }} / V_{\text {crit }}$.

$v_{\phi} \sim 1 / r^{0.5}$ (Keplerian). The above velocity information (principally the small radial velocity) indicates the disk must be rotationally supported.

The theoretical evidence the disk must be Keplerian is based on the suggestion that the $V / R$ variations arise from a precessing one-armed density wave in the disk (Okazaki 1991; Papaloizou et al. 1992). Models of the line emission produced by such a density wave appear to be consistent with observations (Okazaki 1996; Hummel \& Hanuschik 1997), and there are observational correlations indicating a prograde precession of the density wave (Telting et al. 1994; Mennickent, Sterken, \& Vogt 1997). Theoretically, models of a precessing density wave require that the disk be Keplerian (Kato 1983; Okazaki, these proc.).

\subsection{Disk Entrainment}

Given that the disk is Keplerian, what constraints can we place on its origin? The stellar wind blows across the surface of the disk at speeds $v_{w} \sim 1000 \mathrm{~km} \mathrm{~s}^{-1}$. The large velocity shear between the wind and disk causes entrainment of the disk material. Studies of entrainment by astrophysical jets (Cantó \& Raga 1991) suggest the disk entrainment rate is

$$
d \dot{M}_{d} / d r=2 \pi r \rho_{d} \epsilon c_{s},
$$

where the entrainment efficiency $\epsilon=0.01$ (Cantó \& Raga 1991), $\rho_{d}$ is the disk density, and $c_{s}$ is the sound speed.

Interior to the WCD stagnation radius, material falls back onto the star; exterior it flows outward (OCB). Initially, a hole develops in the disk and the hole grows inward and outward. Figure 3 shows the disk clearing time as a function of radius. According to Cantó \& Raga, the flow speed in the boundary layer is $v_{w} / 2$. Balancing momentum, the disk mass-loss rate, $\dot{M}_{d}$, is roughly equal to the wind mass-loss rate through the WCD. Since flow through the WCD can be a sizable fraction of the total stellar mass-loss, $\dot{M}_{*}$, we find $\dot{M}_{d} \sim \dot{M}_{*}$. This implies that the disk will be entirely removed on the disk clearing time scale,

$$
t_{\text {clear }}=M_{d} / \dot{M}_{*} \text {. }
$$


Current estimates of the disk density and its radial structure are provided by measurements of the IR excess (Waters 1986; Waters, Coté, \& Lamers 1987). Based on the amplitude and slope of the free-free emission, they find the disk density $\rho=\rho_{0}(R / r)^{3}$, with $\left(\rho_{0} \sim 10^{-11} \mathrm{gm} \mathrm{cm}^{-3}\right)$. From this density, we obtain a disk mass $M_{d}=10^{-9} M_{\odot}$. We also know that Be stars have a stellar wind with mass-loss rates $\dot{M}_{*}=10^{-10}-10^{-9} M_{\odot} \mathrm{yr}^{-1}$ (Snow 1981, Prinja 1989). The implication is that the Keplerian disk cannot survive for more than about 1-10 years. We conclude that for a disk to remain around a Be star, it must be continually fed at a rate roughly equal to the stellar mass-loss rate, and if its source of material is shut off, it will dissipate in about 1-10 years.

Interestingly, this clearing rate is about the same as the rate observed for X Per (Telting et al. 1998). Telting et al. also find that the length of time to rebuild the disk is about the same as the time to remove it, which requires a mass injection rate into the disk that is comparable to the stellar mass-loss rate. This implies that the source of material for the disk is probably linked to the stellar wind; i.e., somehow the wind feeds the disk.

\subsection{Feeding the Disk}

The WCD mechanism is one possible way to feed the disk. The problem is that the basic model does not add angular momentum. Since Be stars rotate on average at $70 \%$ of critical (Porter 1996), we must add about $100-400 \mathrm{~km} \mathrm{~s}^{-1}$ to the $\phi$-component of the velocity. As noted earlier, adding magnetic fields (of about 10-100 Gauss) to the WCD model might be one possibility (see the results of Chen \& Marlborough 1992). A second suggestion uses non-radial pulsations (Osaki 1986). However since the pulsation amplitude must be less than the sound speed (typically $\lesssim 15 \mathrm{~km} \mathrm{~s}^{-1}$ ), it seems unlikely that NRPs could add the necessary velocity. Another novel idea uses the gravitational tidal torque produced by a binary companion (Lee 1993). Lee finds that when the binary period is in resonance with a low order $g$-mode, sufficient angular momentum can be added to spin-up the outer layers of the star. Unfortunately these specialized conditions make it unlikely that this mechanism can explain all Be stars.

A fourth candidate might be "explosive" events - something like stellar flares (Kroll and Hanuschik 1997). Suppose there is a point-like ejection of material in all directions. Some material will be thrown forward into orbit around the star; some will be thrown backward and return to the star. Kroll (1998) has performed an SPH-simulation of such an event. Initially the material that goes into orbit produces rapid $V / R$ variability. However after a few orbital periods it spreads into a thin ring (owing to orbital phase mixing). Viscosity then causes it spread inward and outward on the viscous timescale $\left(t_{\mathrm{vis}}=r^{2} / \nu \sim\right.$ 100 days), eventually merging with the disk. This model is qualitatively similar to the ring ejection model of Huang (1976). It also produces behavior that is quite similar to that observed by Rivinius et al. (1998a, 1998b) during the recent outbursts of $\mu$ Cen, so this flare scenario is quite intriguing.

\section{Cluster Clues}

The last set of clues for disk formation mechanisms comes from studies of Be stars in low metallicity clusters (Grebel, Richtler, \& de Boer 1992; Grebel 1997; Maza- 
lli et al. 1996; Keller, Wood, \& Bessell 1999). Maeder, Grebel, \& Mermilliod (1999) find that the frequency of Be stars increases as the metallicity decreases. It is known that the terminal speed decreases as the metallicity decreases (Abbott 1982; Garmany \& Fitzpatrick 1988). According to the WCD model, a lower terminal speed will decrease the disk formation threshold, qualitatively explaining the increase in frequency of Be stars at low metallicity. Similarly, the ion decoupling radius decreases as the metallicity decreases (Porter, private communication), which also increases the frequency of Be stars. Note however that Maeder et al. conclude that the WCD model cannot explain the increased frequency of Be stars because the decrease in metallicity will shut off the wind (on the other hand there is no observational evidence for lower mass-loss rates in the Magellanic clouds, Garmany \& Fitzpatrick 1988).

More recently, the suggestion that the frequency of Be stars is higher for low metallicity has been challenged by Keller et al. (1999). They suggest that the real effect is one of age. They find that the frequency of Be stars is a maximum at the main-sequence turnoff (age $\sim 10 \mathrm{Myr}$ ). This revives the suggestion that $\mathrm{Be}$ stars tend to be slightly evolved. Why is uncertain, but MacGregor \& Cassinelli (these proc.) proposed that magnetic loops generated at the outer boundary of the convective core could rise to $80 \%$ of the stellar radius, which is $99.9 \%$ of the stellar mass. The envelope mass above this stall-radius is $M_{\text {env }}=0.01 M_{\odot}$. For a mass-loss rate of $10^{-9} M_{\odot} \mathrm{yr}^{-1}$, the time required to remove the outer atmosphere, exposing the magnetic fields, would be $t_{\mathrm{mag}}=M_{\mathrm{env}} / \dot{M}_{*}=10^{7} \mathrm{yr}$, which is the same as the age when the frequency of Be stars is a maximum. This suggests it may be possible to have surface magnetic fields that could be responsible for flaring activity or for use in transporting the angular momentum necessary to make a Keplerian disk.

\section{Conclusion}

Now that observations indicate that the disks around Be stars are Keplerian, the primary challenge for theoretical models is to determine how to continually feed the disk and how to transport angular momentum to the material entering the disk. One mechanism for feeding the disk is the WCD model. Although nonradial forces may inhibit the wind-compression, there are several likely scenarios that may turn off the non-radial forces (prime candidates are clumping in the wind and ion decoupling). However, the WCD model must be modified to add a mechanism for transporting angular momentum if it is to be successful. One possibility is adding magnetic fields, which could be generated at the edge of the convective core (and rise to the stellar surface after sufficient stellar mass-loss). A second way to add angular momentum is "explosive" mass ejection. This scenario produces phenomena similar to those observed in the outbursts of $\mu$ Cen. Why such events would be linked to multiple NRPs adding in phase is unclear, but perhaps we should take that as a clue that maybe NRPs somehow trigger flaring activity. Finally we note that disk entrainment provides a natural source of variability and a timescale for disk dissipation of about 1-10 years.

Acknowledgments. This work has been supported under NASA grant NAG5-3248 to the University of Toledo. 


\section{References}

Abbott, D.C. 1982, ApJ, 259, 282

Bjorkman, J.E. 1994, in IAU Symp., Pulsation, Rotation, and Mass Loss in Early-Type Stars, ed. L.A. Balona, H.F. Henrichs, \& J.-M. leContel (Dordrecht: Kluwer), 455

Bjorkman, J.E. 1997, in Stellar Atmospheres: Theory \& Observations, Springer Lecture Notes in Physics, Vol. 497, ed. J.-P. deGreve, R. Blomme, \& H. Hensberge (Berlin: Springer), 239

Bjorkman, J.E., Abbott, B.P., \& MacFarlane, J.J. 2000 (in prep.)

Bjorkman, J.E., \& Cassinelli, J.P. 1993, ApJ, 409, 429

Cantó, J., Raga, A.C. 1991, ApJ, 372, 646

Castor, J.I., Abbott, D.C., \& Klein, R.I. 1975, ApJ, 195, 157 (CAK)

Chen, H., \& Marlborough, J.M. 1992, ApJ, 397, 242

Chen, H., \& Marlborough, J.M. 1994, ApJ, 427, 1005

Chen, H., Marlborough, J.M., \& Waters, L.B.F.M. 1992, ApJ, 384, 605

de Araújo, F.X. 1995, A\&A, 298, 179

de Araújo, F.X., \& de Freitas Pacheco, J.A. 1989, MNRAS, 241, 543

de Araújo, F.X., de Freitas Pacheco, J.A., \& Petrini, D. 1994, MNRAS, 267, 501

Frank, J., King, A.R., \& Raine, D.J. 1985, Accretion Power in Astrophysics (Cambridge: Cambridge Univ. Press)

Friend, D.B., \& Abbott, D.C. 1986, ApJ, 311, 701

Garmany, C.D., \& Fitzpatrick, E.L. 1988, ApJ, 332, 711

Grebel, E.K. 1997, A\&A, 317, 448

Grebel, E.K., Richtler, T., \& de Boer, K.S. 1992, A\&A, 254, L5

Hanuschik, R.W. 1988, A\&A, 190, 187

Hanuschik, R.W. 1995, A\&A, 295, 423

Hanuschik, R.W. 1996 A\&A, 308, 170

Hanuschik, R.W., Kozok, J.R., \& Kaiser, D. 1988, A\&A, 189, 147

Harmanec, P. \& Kříż, S. 1976, in IAU Symp. 70, Be and Shell Stars, ed. A. Slettebak, (Dordrecht: Reidel), 3

Huang, S.-S. 1976, PASP, 88, 448

Hummel, W., \& Hanuschik, R.W. 1997, A\&A, 320, 852

Ignace, R., Cassinelli, J.P., \& Bjorkman, J.E. 1996, ApJ, 459, 671

Kato, S. 1983, PASJ, 35, 249

Keller, S.C., Wood, P.R., \& Bessell, M.S. 1999, A\&AS, 134, 489

Koninx, J.P.M. 1992, in ASP Conf. Ser. 22, Nonisotropic and Variable Outflows from Stars, ed. L. Drissen, C. Leitherer, \& A. Nota (San Francisco: ASP), 98

Koninx, J.P.M., \& Hearn, A.G. 1992, A\&A, 263, 208

Kroll, P., \& Hanuschik, R.W. 1997 in IAU Colloq. 163, ASP Conf. Ser. 121, Accretion Phenomena and Related Outflows, ed. D.T. Wickramasinghe, G.V. Bicknell, \& L. Ferrario (San Francisco: ASP), 494 
Kroll, P. 1998, Ph.D. thesis

Lamers, H.J.G.L.M., \& Pauldrach, A.W.A. 1991, A\&A, 244, L5

Lee, U. 1993, ApJ, 417, 697

Lee, U., Saio, H., \& Osaki, Y. 1991, MNRAS, 250, 432

MacFarlane, J.J., et al. 1993, ApJ, 419, 813

Maeder, A., Grebel, E.K., \& Mermilliod, J.-C. 1999, A\&A, 346, 459

Maeder, A., \& Meynet, G. 1989, A\&A, 210, 155

Marlborough, J.M., Chen, H., \& Waters, L.B.F.M. 1993, ApJ, 408, 646

Mazalli, P.A., et al. 1996, A\&A, 316, 173

Mennickent, R.E., Sterken, C., \& Vogt, N. 1997, A\&A, 326, 1167

Mennickent, R.E., et al. 1994, A\&AS, 106, 427

Okazaki, A.T. 1991, PASJ, 43, 75

Okazaki, A.T. 1996, PASJ, 48, 305

Osaki, Y. 1986 PASP, 98, 32

Owocki, S.P., Cranmer, S.R., \& Blondin, J.M. 1994, ApJ, 424, 887 (OCB)

Owocki, S.P., Cranmer, S.R., \& Gayley, K.G. 1996, ApJ, 472, L115 (OCG)

Papaloizou, J.C., Savonije, G.J., \& Henrichs, H.F. 1992, A\&A, 265, L45

Pauldrach, A.W.A, \& Puls, J. 1990, A\&A, 237, 409

Poe, C.H. 1987, Ph.D. thesis (Univ. Wisconsin)

Poe, C.H., \& Friend, D.B. 1986, ApJ, 311, 317

Pols, O.R., et al. 1991, A\&A, 241, 419

Porter, J.M. 1996, MNRAS, 280, L31

Porter, J.M. 1997, A\&A, 324, 597

Porter, J.M., \& Drew, J.E. 1995, A\&A, 296, 761

Porter, J.M., \& Skouza, B.A. 1999, A\&A, 344, 205

Prinja, R.K. 1989, MNRAS, 241, 721

Quirrenbach, A., et al. 1997, ApJ, 479, 477

Rivinius, Th., et al. 1998a, A\&A, 333, 125

Rivinius, Th., et al. 1998b, A\&A, 336, 177

Shakura, N.I., Sunyaev, R.A. 1973, A\&A, 24, 337

Snow, T.P. 1981, ApJ, 251, 139

Springmann, U.W.E., \& Pauldrach, A.W.A. 1992, A\&A, 262, 515

Telting, J.H., et al. 1994, A\&A, 288, 558

Telting, J.H., et al. 1998, MNRAS, 296, 785

Van Bever, J., \& Vanbeveren, D. 1997, A\&A, 322, 116

von Zeipel, H. 1924, MNRAS, 84, 665

Waters, L.B.F.M. 1986, A\&A, 162, 121

Waters, L.B.F.M., Coté, J., \& Lamers, H.J.G.L.M. 1987, A\&A, 185, 206

Wood, K., Bjorkman, K.S., \& Bjorkman, J.E. 1997, ApJ, 477, 926

Zaal, P.A., et al. 1997, A\&A, 326, 237 


\section{Discussion}

Ph. Stee: You said that 1-D radiative wind models fail to reproduce terminal speeds $\lesssim 100 \mathrm{~km} \mathrm{~s}^{-1}$. This is not true; at least the Lamers \& Pauldrach (1991) bistability model is able to reproduce a velocity of $1000 \mathrm{~km} \mathrm{~s}^{-1}$ in the pole vs. $100 \mathrm{~km} \mathrm{~s}^{-1}$ in the equator. I think that the WCD model is not able to produce such a low equatorial velocity, which is an additional difficulty.

J. Bjorkman: WCD models typically give a disk terminal speed of about $200-400 \mathrm{~km} \mathrm{~s}^{-1}$ (OCB), which is much too large. Although, the bistability model gives an equatorial terminal speed of about $200 \mathrm{~km} \mathrm{~s}^{-1}$, that value is for $\mathrm{B}[\mathrm{e}]$ supergiants, which have significaritly lower surface gravities $(\log g \sim$ 2). The bistability jump reduces the ratio $v_{\infty} / v_{\text {esc }}$ from 2.7 to 1.3 (about a factor of 2). For Be stars, the polar escape speed is about $900 \mathrm{~km} \mathrm{~s}^{-1}$, so the predicted terminal speed on the low (equatorial) side of the jump would be about $1200 \mathrm{~km} \mathrm{~s}^{-1}$. Even if we lower the polar terminal speed to the observed value $\left(\sim 1000 \mathrm{~km} \mathrm{~s}^{-1}\right)$, the equatorial terminal speed still would be $\sim 500 \mathrm{~km} \mathrm{~s}^{-1}$. Unless the star is rotating very close to critical (so that the equatorial surface gravity is significantly reduced), it will be very difficult for the bistability model to have an equatorial terminal speed $\lesssim 100 \mathrm{~km} \mathrm{~s}^{-1}$.

S. Owocki: Your estimate of the disk entrainment rate implies that every gram of wind must bring with it a gram of disk material. Given that only a small fraction of the wind comes in contact with the disk boundary layer, doesn't that seem to be an overestimate?

J. Bjorkman: Perhaps, but this model supposes that the Keplerian disk is being replaced by a WCD. That is why a hole first develops at the WCD stagnation radius (where most of the wind is initially deposited). Then interior to the hole, the infalling WCD material carries that portion of the Keplerian disk inward, while the outflowing WCD material entrains the outer part of the Keplerian disk. Since a large fraction of the total stellar mass-loss passes through the WCD, it also subsequently passes over the surface of the Keplerian disk. Thus I would argue that the relevant mass-loss rate to use for the entrainment source is the mass-loss passing through the WCD.

H. Henrichs: I would lower your estimate for the magnetic field strength required to eject material into Keplerian orbit. First, we have seen in $\beta$ Cep that a field of $100 \mathrm{G}$ has a very drastic effect on the wind, producing a magnetically controlled wind with an estimated Alfvén radius of $10 R_{*}$. Second, such a strong field gives a rapid spin-down timescale, which is incompatible with the observed high rotational velocities.

J. Cassinelli: You showed some $\mathrm{Br} \alpha$ line profiles that agreed with the densities produced by the WCD model. In Okazaki's talk, we heard that Keplerian disks are required to match $\mathrm{H} \alpha$ observations. Does this mean that some stars have WCDs and others have Keplerian disks; i.e., is there an angular momentum problem for all Be stars, or only for some fraction of them?

J. Bjorkman: Although these stars have double-peaked $\mathrm{Br} \alpha$ emission, they do not have Balmer line emission or a detectable IR excess, so they are not classified as classical Be stars. Nonetheless, it appears they have a circumstellar disk. The suggestion is that this weak disk may be the WCD; whereas, classical Be stars appear to have Keplerian disks. 